\title{
Improvement of the Vertical Handover Decision and Quality of Service in Heterogeneous Wireless Networks using Software Defined Network
}

\author{
Fatima Laassiri ${ }^{1, \text { a }}$, Mohamed Moughit ${ }^{2, \mathrm{~b}}$, Noureddine Idboufker ${ }^{3, \mathrm{c}}$ \\ ${ }^{\mathrm{a}, \mathrm{b}}$ IR2M Laboratory, FST, Univ Hassan UH1- Settat, Morocco \\ ${ }^{\mathrm{b}}$ EEA\&TI Laboratory, FST, Univ Hassan, Mohammedia, Morocco \\ ${ }^{b}$ National Schools of Applied Sciences Khouribga, Univ Hassan 1, UH1 - Settat, Morocco \\ ${ }^{c}$ National School of Applied Sciences, Univ Cadi Ayyad Marrakech, Morocco
}

\begin{abstract}
The development of wireless networks brings people great convenience. More state-of-the-art communication protocols of wireless networks are getting mature. People attach more importance to the connections between heterogeneous wireless networks as well as the transparency of transmission quality guarantees. Wireless networks are an emerging solution based on users' access to information and services, regardless of their geographic location. The success of these networks in recent years has generated great interest from individuals, businesses and industry. Although there are several access technologies available to the user such as IEEE standards (802.11, and 802.16).SDN is a new network paradigm used to simplify network management, reducing the complexity of network technology. The following work aims to expose a simulation implemented under OMNeT 4.6 ++, to improve the Handover performance between two technologies WiFi and WiMAX. This paper proposes a decision algorithm for a heterogeneous vertical handover between WiFi access points and WiMAX network. The inputs to the algorithm are WiFi RSS, bit rate, jitter, and estimated TCP end-to-end delay.
\end{abstract}

Keywords-Heterogeneous network; vertical handover; WiMAX; WiFi; IEEE 802.16; IEEE 802.11; OMNeT4.6

\section{INTRODUCTION}

Recently, the Software-Defined Network (SDN) plays an important role because of its flexibility and ease of transportation. Worldwide Interoperability for Microwave Access (WiMAX)[1] or Global Interoperability for Microwave Access is the promising Fourth Generation (4G)[2] network to meet the needs of customers. It is a telecommunication technology that it provides softwaredefined data for several distances from a point-to-point links to all cell-type accesses, that it allows the connection between mobile and fixed networks. The coverage area of WiMAX is high compared to other technologies; it offers good support and good stability.

Wireless Fidelity (WiFi) [3] provides broadband connectivity for local area networks, while WiMAX provides broadband coverage in the metropolitan area with guaranteed quality of service. WiFi access points usually offer free access to good time users, while WiMAX overlay networks offer paid access to users. It is therefore necessary to provide $\mathrm{WiFi}$ connectivity for such a long time and to allow a roaming mobile device to switch to a superimposed WiMAX network only when the WiFi services are out of range or when its quality of service becomes unacceptable, which means that the WiMAX coverage is supposed to be always available and that the mobile terminal has to switch between WiFi depending on the availability of $\mathrm{WiFi}$ access points.

It offers another aspect of how conventional remote systems have been described, due to the effects of innovation on the public and its impact on the environnent. The mechanical points of interest of WiMAX are central points of unprecedented WiMAX radio change, it works in the field of security and quality, and it has an open access base focused on IP access. This innovation can be used for various applications, WiMAX is a scalable remote correspondence system capable of providing high-speed remote access with high data rate of $4 \mathrm{G}$ over a long separation in a point-tomultipoint and visible or an unobservable path condition.

This article presents an evaluation and improvement in Handover vertical [4] performance between $\mathrm{WiFi}$ and WiMAX via a decision algorithm for the heterogeneous transfer between WiFi access points and a WiMAX networks. The inputs to the algorithm are WiFi Received Signal Strength (RSS)[5], bit rate, jitter, and estimated Transmission Control Protocol (TCP)[6] end-to-end delay.

\section{STATE OF THE ART}

Wireless networks at the forefront of microwave technology and useful in broadband access, as a central innovation for the IEEE 802.16 reference group, are advancing in $4 \mathrm{G}$ discovery. With the current presentation of portability management systems in the IEEE 802.16e standard, it is currently competing with current and future ages of remote advances to provide ubiquitous recording arrangements. Nevertheless, the establishment of a decent versatile structure depends to a large extent on the ability to make quick and consistent transfers regardless of the situation of the building being sent. Since the IEEE has characterized the mobile WiMAX MAC layer transfer. (IEEE 802.16e) administration structure and the WiFi MAC layer (IEEE 802.11), the WiMAX Forum and WiMAX Network Workgroup (NWG) [7] are processing the improvement of the upper layers. That is, the path to commercialization of an 
undeniable structure of versatility, that it is in charge of investigating the difficulties. This focuses on potential research issues related to the transfer into the current and future wireless portability structure. An examination of these issues in the MAC, Network and Cross-Layer situations is presented alongside the exchange of distinctive answers to these difficulties.

Subscriber Station (SS) [8] gives the link to WiMAX. Many websites are used to provide this method, but this is not a complete view of the tools available as certified that they are set in mobile Internet devices and various private labelled tools, laptops.

It assembles Wi-Fi activities because of the same Wireless Network. It also gives network connectivity to businesses and at home without the help of external devices. For this, it uses WiMAX carriers. It is scaled a few kilometers. It varies at the scale of the city. It is given with a subscriber unit. It helps the customer to connect to the Internet and other accesses.

According to research carried out by the "Mojtaba Seyedzadegan"[9] on the overview of wireless networks, its architecture deals with the supply of data. It is entirely based on IEEE 802.16. It supports two things one is the alternative broadband cable and the other is DSL.

On the basis of the existing works of the following researchers: Marceau Coupechoux, Jean-Marc Kifel, Philippe Godlewski that they accumulate the wireless ones in what they follow

- The users arrive at random times.

- The location of the users is taken into account.

- The choice of the network takes place at the arrival of the user.

- The decision is made by a dynamic programming algorithm. Limitations

- Digital resolution is limited by the size of the problem.

- The network cells are concentric.

- The following researchers: Srinivas Shakkotai, Eitan Altman, Anurag Kumare, they present the following states:

- The user population is constant over time.

- Users can connect to multiple WiFi networks simultaneously (Multi-homing).

- The model is macroscopic: The populations follow a dynamic that converges to a state that maximizes the sum of user flows. Limitations

- The cells belong to the same technology (Wi-Fi).

- The algorithm to obtain the dynamics is not provided.

\section{PROBLEM AND SOLUTIONS}

Wi-Fi and WiMAX refer to certain types of Wireless Networks Wireless Local Area Network (WLAN)[10] and Wireless Metropolitan Area Network (WMAN)[10], using
802.11 [11] and 802.16[12] specifications. They are widely used by businesses.

Because of the increasing demand, users ubiquitous access to wireless services, which he led to the deployment of a forced use of this wireless technology such as Wi-Fi, it offers a level of quality in the range, but the problem is that the number of devices is increasing, which reduces the performance of travel time Handover and QoS[13] for all these reasons, this work proposes a solution for the improvement of vertical Handover by the creation of an algorithm which is implemented at the level of SDN controller, to better optimize the performances.

OpenFlow [14] is a first attempt to develop a wireless SDN platform with Stanford University. It separates the control plane from the data path and produces slices of network using FlowVisor [15] to isolate the different flows.

The underlying infrastructure is configured with SNMPVisor, a line interface command for configuring data path elements with Simple Network Management Protocol (SNMP) [16]. In other words, OpenFlow allows multiple different experiences and services to run simultaneously on a physical network.

Researchers are currently looking to implement the OpenFlow to WiFi access points and WiMAX base stations for traffic control and use a network controller, to communicate with OpenFlow devices and to provide global network views. FlowVisor can be considered a transparent proxy [17] for OpenFlow. It slices the network by selectively rewriting or dropping OpenFlow messages to delegate control of different streams with different controllers. The structure of OpenFlow is shown in figure 1 to separate the traffic of different users with multiple transmission policies.

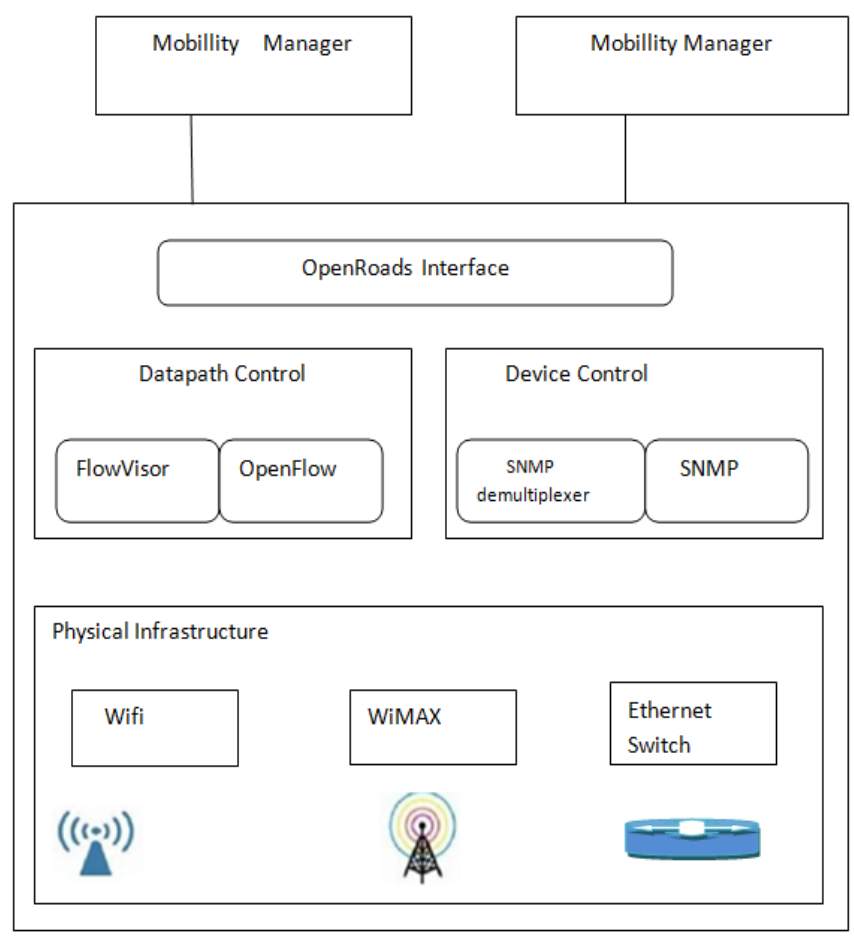

Fig. 1. Openflow Architecture. 
Currently, the radio access network uses distributed algorithms to manage the limited spectrums and transmit Handover. While the decision in a little environment with few base stations could be simple to make, it would be harder to quickly pick the best candidate for deployment ladders. To manage growing mobile base station traffic, this research proposes that Software Defined Wireless Network (SDWN) [18] is a centralized system for software-defined radio access networks, to effectively perform transfers and allocate spectrum resources as well as to set spectrum values.

In this articlee, we propose a decision algorithm for a heterogeneous vertical Handover between WiFi access points and a WiMAX network capitalize. The inputs to the algorithm are WiFi RSS, bit rates, jitter, and estimated TCP end-to-end delay.

WiFi provides broadband connectivity for local area networks, while the WiMAX network provides broadband coverage in the metropolitan area with guaranteed quality of service. WiFi access points typically offer free access to goodtrack users, while WiMAX overlay networks offer paid access to users. It is therefore necessary to provide WiFi connectivity for as long as possible and to allow a roaming mobile device to switch over to a WiMAX accumulate network only when WiFi services are out of range or when its quality of service becomes unacceptable, which it means that WiMAX coverage is supposed to be always available and that the mobile terminal has to switch between $\mathrm{WiFi}$ depending on the availability of WiFi access points.

\section{CONTRIBUTION}

The following algorithm (Figure 2) shows the calculation steps implemented when approaching a mobile terminal, and when moving away from a $\mathrm{WiFi}$ access point, in both cases, the flow samples WiFi feeds are measured at regular time intervals and a moving average value Media RSS (MRSS) [19] is calculated for each RSS sample at the SDN controller level.

Considering first the case where the mobile terminal approaches the WiFi access point, if the calculated value of the MRSS is less than or equal to the sensitivity of the receiver, it means that the mobile terminal has already left the $\mathrm{WiFi}$ reception area. In this case, the WiFi Handover to WiMAX is immediately launched and centralized by the SDN controller. On the other hand, the MRSS value exceeds the property of the receiver, the position and the current speed of the mobile are calculated. These two values make it possible to determine the remaining time before the mobile terminal crosses the $\mathrm{WiFi}$ reception boundary. If this time exceeds the specified end-to-end TCP handshake delay, a prerelease routine is initiated by the SDN controller to ensure transparent Handover to the WiMAX network. This Handover pre-break routine has been adopted to ensure that Internet connectivity is maintained throughout the Handover process.

Considering thereafter, the case where the mobile terminal moves away from the $\mathrm{WiFi}$ access point. If the calculated value of the MRSS is greater than or equal to the sensitivity of the receiver, it means that the mobile terminal is already in the WiFi reception area. In this case, the WiFi WiMAX Handover is immediately launched. If, on the other hand, the MRSS value is lower than the sensitivity of the receiver, the current position and speed of the mobile are calculated. These two values make it possible to determine the remaining time before the mobile terminal enters the $\mathrm{WiFi}$ reception area. If this time exceeds the specified end-to-end TCP transfer latency, the transfer routine before the WiFi network cutoff is initiated. This routine makes it possible to provide connectivity to the Internet via the WiFi network as soon as the mobile terminal reaches the limit of its reception area. This ensures that the mobile terminal maximizes connectivity via the WiFi network.

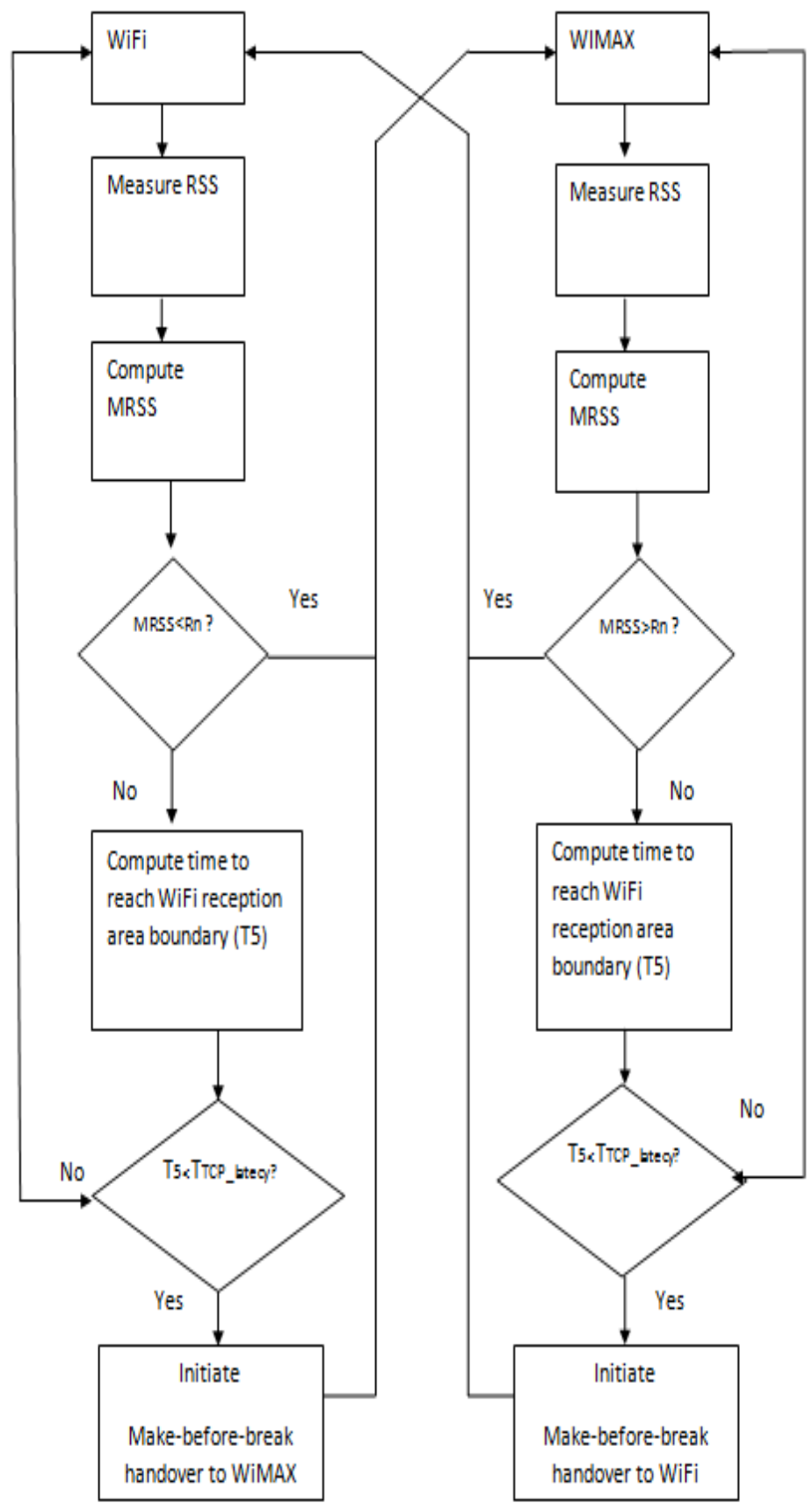

Fig. 2. The Flowchart of the Proposed Vertical Handover Algorithm. 


\section{DIFFERENCE BETWEEN WIMAX AND WIFI}

The following table presents a difference between $\mathrm{WiFi}$ technology and WiMAX.

TABLE I. DIFFERENCE BETWEEN WIMAX AND WIFI

\begin{tabular}{|l|l|l|}
\hline Parameter & WiFi (802.11n) & WiMAX(802.16e) \\
\hline Method of access & OFDM & OFDMA \\
\hline Frequency band & 2.4 or 5 GHZ & $\begin{array}{l}2.3-2.4,2.496- \\
2.69,3-3.8 \\
\text { GHZ }\end{array}$ \\
\hline $\begin{array}{l}\text { Max Throughput } \\
\text { Downlink Uplink }\end{array}$ & $540 \mathrm{Mbps}$ & $75 \mathrm{Mbps} 25 \mathrm{Mbps}$ \\
\hline Cellule coverage & $400 \mathrm{~m}$ & $2-7 \mathrm{Km}$ \\
\hline Cellule capacity & 32 & $100-200$ \\
\hline $\begin{array}{l}\text { Supported } \\
\text { mobility }\end{array}$ & $4 \mathrm{Km} / \mathrm{h}$ & $120 \mathrm{Km} / \mathrm{h}$ \\
\hline Service cost & Low & High \\
\hline $\begin{array}{l}\text { Energy } \\
\text { consumption }\end{array}$ & Medium & High \\
\hline $\begin{array}{l}\text { Security } \\
\text { Quality of service }\end{array}$ & Yes & High \\
\hline
\end{tabular}

VI. WIRELESS CONNECTION MODES

Two modes exist

The infrastructure mode is the most common. The machines communicate via an access point that it is only a kind of WiFi HUB.

The Ad Hoc[20] mode is more particular, since it makes it possible to dispense with the access point. Security possibilities in this mode are, however, almost non-existent. This mode should be avoided.

\section{METHODS AND IMPLEMENTATION OF WIMAX AND WIFI USING SDN}

The transparent Handover algorithm has been evaluated both in the case where a mobile terminal approach one WiFi access point to another. In each case, simulations were performed for typical speeds that the mobile terminal could encounter. The typical handover latency between $\mathrm{WiFi}$ and WiMAX for an end-to-end TCP connection varies from 450 ms to 1 second.

The distance from the $\mathrm{WiFi}$ reception limit at which the transfer process started has been recorded for transfer delays ranging from $10 \mathrm{~ms}$ to $1000 \mathrm{~ms}$, this distance was the minimum distance, determined by our algorithm, for the Handover to be done transparently. The maximum reception distance of the WiFi access point is obtained using the path loss equation (1)

Plossd $\mathrm{B}=$

$20 \log 10(4 \pi / \lambda)+10 \rho \log 10 \mathrm{~d}$

Where PlossdB is the signal loss in $\mathrm{dB}$ between the transmitter and the receiver; $\lambda$ is the wavelength of the $\mathrm{WiFi}$ signal

$\rho$ is a path loss constant

$\mathrm{d}$ is the distance between the transmitter and the receiver.

The maximum reception distance for $\mathrm{WiFi}$ reception occurs when the path loss calculated by equation (1) above is equal to the difference between transmit power and receiver sensitivity. This algorithm uses a $\mathrm{WiFi}$ transmission power of $+17 \mathrm{dBm}$, an outside path loss factor of 2 and a receiver sensitivity of $-76 \mathrm{dBm}$. Substituting these values in Equation (1) gives a maximum receiving distance of 994 meters.

This work consists of two vertical Handover scenarios between WiFi and WiMAX with and without SDN including

Scenario 1(Table 2, 3, 4): Vertical handover between WiFi and WiMAX without SDN: 3 AP, 7 WiFi Clients, 7 WiMAX Clients, and 3 BS. (Figure 3)

Scenario 2 (Table 2, 3, 4) Vertical handover between WiFi and WiMAX with SDN: 3 APs, 7 WiFi Clients, 7 WiMAX Clients, and 3 BS. (Figure 4)

The trajectory is defined as follows:

For the WiMAX scenario, the mobility speed is set at 20 $\mathrm{km} /$ hour;

Two meters per second in a WiFi network;

The WiFi $802.11 \mathrm{~b}$ standard with a bandwidth of $2 \mathrm{Mb}$ was used to reach a theoretical coverage area of 400 meters.

The simulation parameters used in $\mathrm{WiFi}$ scenarios are listed in the table below:

TABLE II. BASE STATION PARAMETERS

\begin{tabular}{|l|l|}
\hline Parameter & Value \\
\hline Antenna Gain & $15 \mathrm{dBi}$ \\
\hline Number of transmitters & SISO \\
\hline Maximal transmission power & $500 \mathrm{~mW}$ \\
\hline PHY profile & OFDM \\
\hline Maximal power density & $-60 \mathrm{dBm}$ \\
\hline Minimal power density & $-110 \mathrm{dBm}$ \\
\hline The resource retention time & $200 \mathrm{msec}$ \\
\hline
\end{tabular}

TABLE III. ACCESS POINT SETTINGS

\begin{tabular}{|l|l|}
\hline Parameter & Value \\
\hline PHY mode & Direct Sequence \\
\hline Throughput & $2 \mathrm{Mbps}$ \\
\hline Transmission power & $0,005 \mathrm{~W}$ \\
\hline Beacon interval & 0,02 Secs \\
\hline Buffer size & 256 Kilobits \\
\hline
\end{tabular}


TABLE IV. APPLICATION SETTINGS

\begin{tabular}{|l|l|}
\hline Parameter & Value \\
\hline Traffic & VOIP \\
\hline Codec & G729A \\
\hline Voice frames per packet & 1 \\
\hline Traffic generation & $\begin{array}{l}\text { Continuously and infinite (from } \\
\text { the start to end of the simulation) }\end{array}$ \\
\hline
\end{tabular}

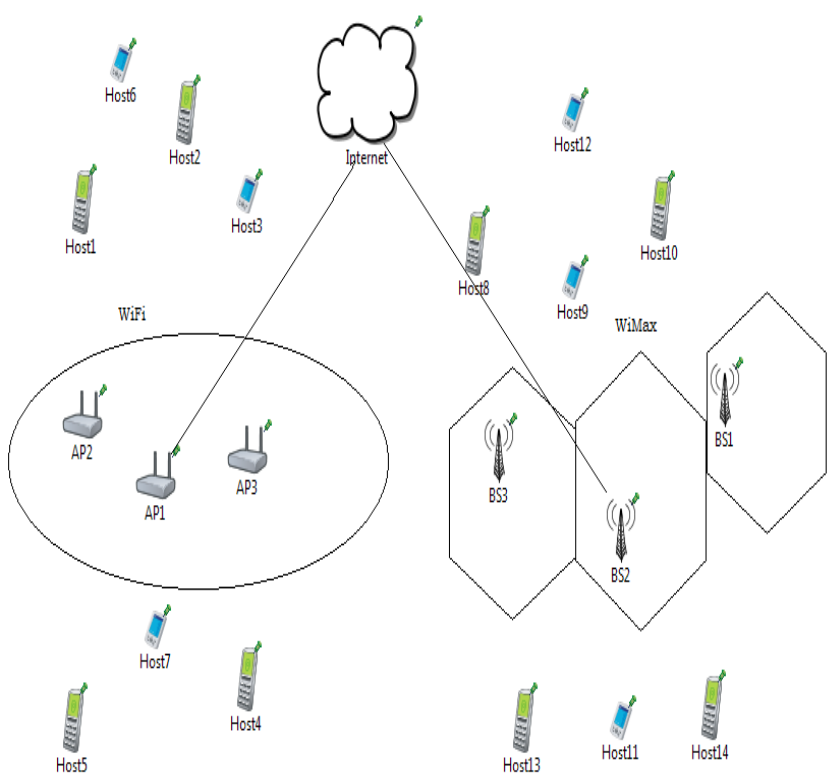

Vertical Handover

Fig. 3. Vertical and Horizontal Handover without SDN.

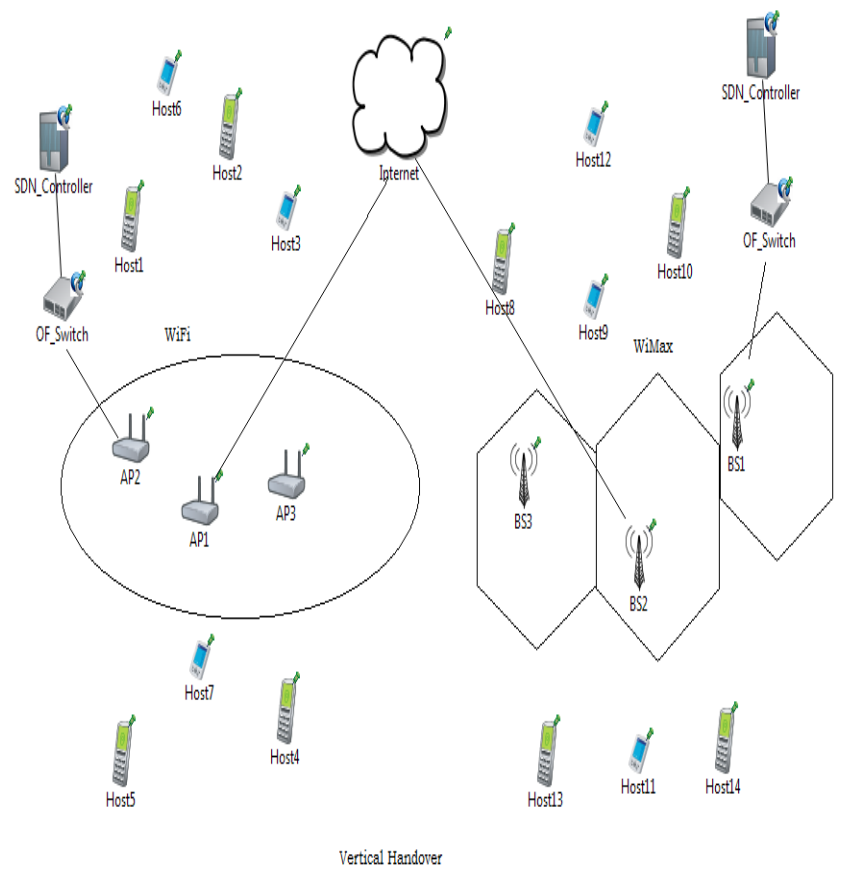

Fig. 4. Vertical and Horizontal Handover with SDN.
In order to take into account the mobility of users, the standard implements a Handover procedure that can be used in the following cases:

When the mobile station MS (Mobile Station) can be taken into account with a better signal quality by another base station (terminal movement, signal attenuation or interference).

When the mobile terminal can be taken into account with a better QoS by another base station (Load balancing [21], admission control, or QoS expectations).

\section{IMPROVEMENT IN HANDOVER}

Reducing the duration of a Handover is one of the goals. This can be done by developing a Handover protocol. Researchers are interested in reducing the duration of Handover by offering a cooperative Handover to prematurely prepare the Handover with the target network. The researchers are looking for a seamless vertical handover. For this, they present a new concept called "Takeover". This is to allow a neighbouring node in the recovery area to process requests from a mobile node that it wants to make a Handover before it can implement it. This is called Cooperative Handover as the nodes help each other with the Handover. This reduces the Handover time: Pre-authentication and preregistration time (Using a mobile IP registration procedure). In addition, a protocol for the Takeover has been developed and applied. Handover's decision is based on the signal quality of the two base stations. On the other hand, the proposed system requires more signalling and also more processing by the neighbouring node. This finally requires operation in mesh mode (Direct communication between terminals).

\section{Mobility Management}

In fixed broadband access, WiMAX distinguishes four types of mobility related to the circumstances of use:

Nomad: In this case, a fixed place is assigned to the user for the use of the services. In order to connect to a different location, the user must make a break or disconnect [22];

Portable: Using nomadic access with a portable device, portability is ensured for the user at the cost of the Handover [23];

Simple mobility: Almost uninterrupted Handovers are made with users who can reach a speed of $60 \mathrm{~km} / \mathrm{h}$ in very short time intervals [24];

Full mobility: The user can move up to a speed of $120 \mathrm{~km}$ / h [25].

The Handover is the mechanism that ensures the continuity of the connection of a subscriber's station during its movement of the coverage area from one base station to another.

\section{HANDOVER PROCESS}

The Handover process can be carried out in three phases:

Transfer Information Collection: Also known as System Discovery, the information required to identify the need transfer is collected. In this phase, information from all 
neighbouring networks is collected, and they can also be called the system discovery phase.

Transfer decision: This process finds the appropriate candidate network to which the mobile terminal "MT" can be transferred according to certain decision algorithms.

Transfer execution: Finally, the signalling exchange for the establishment of the new communication path was carried out with the rerouting of data via this path.

Network Selection: The Horizontal Transfer Decision Algorithm includes the strength of the received RSS signal. For the Vertical transfer decision, many criteria can be taken into account, such as: Cost of service, power consumption, mobile device speed, and user preference.

\section{RESULTS AND DISCUSSION}

The following figure (5) shows that WiMAX does not require more delay, compared to $\mathrm{WiFi}$, for $\mathrm{WiFi}$ to WiMAX transfer or vice versa, compared to $\mathrm{WiFi}$, this is quite logical because the WiMAX network was designed primarily to connect a very large number of users with a high bandwidth with a minimum of time.

Figures 6 illustrates the network delay of $\mathrm{WiFi}$ and WiMAX scenarios using SDN In WiMAX, and WiFi, they represent the end-to-end delay of all packets received by WiMAX or WiFi MACs, all nodes of the network, and they transmit to the upper layer.

MN can discover a suitable WiFi network via a requestresponse

Transfer to WiFi based on a number of factors such as the quality of service, power, cost, etc. If this discovery is successful, the mobile initiates the transfer procedures.

After transferring to $\mathrm{WiFi}$, the $\mathrm{MN}$ can choose to set the WiMAX radio to sleep mode. This allows the MN to quickly return to WiMAX in case of $\mathrm{WiFi}$, the coverage is abruptly degraded. Figure 7 shows the process of transfer between WiMAX and WiFi.

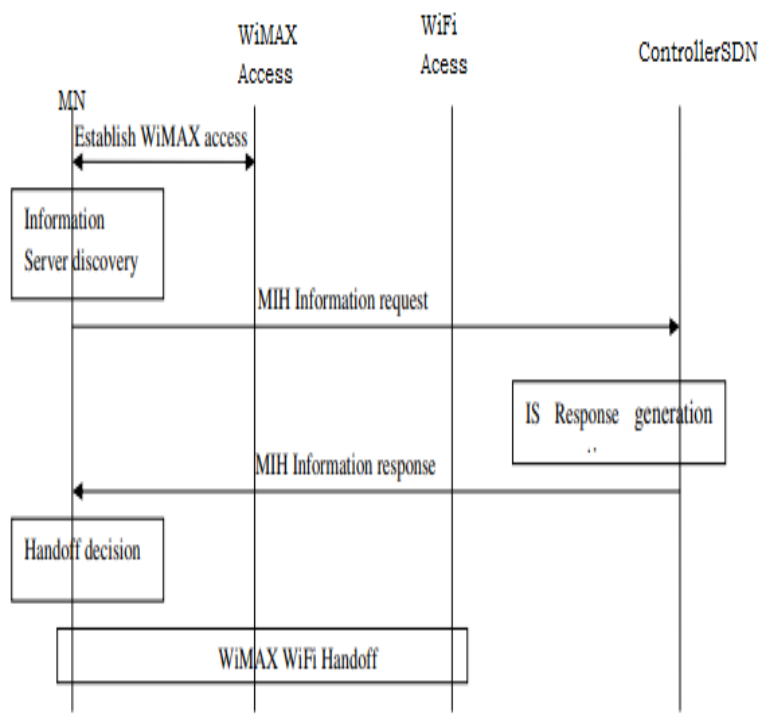

Fig. 5. Handover from WiMAX to WiFi using SDN Controller.

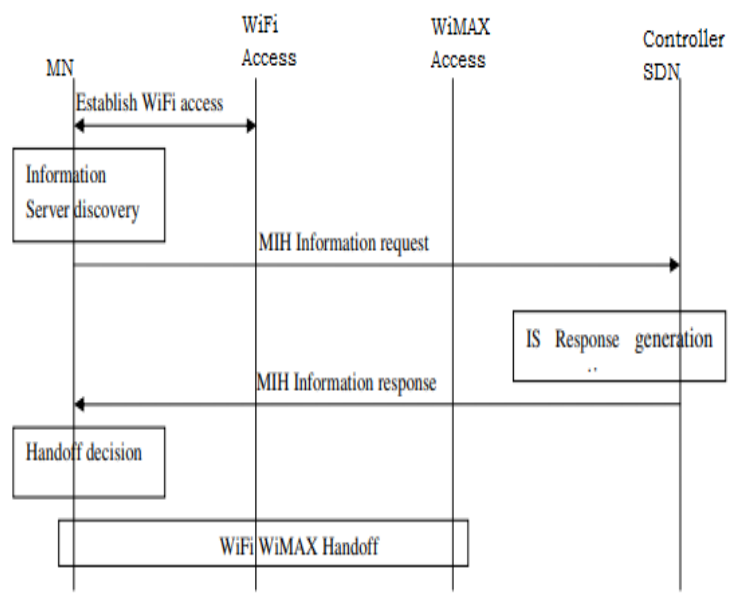

Fig. 6. Handover from WiFi to WiMAX using SDN Controller.

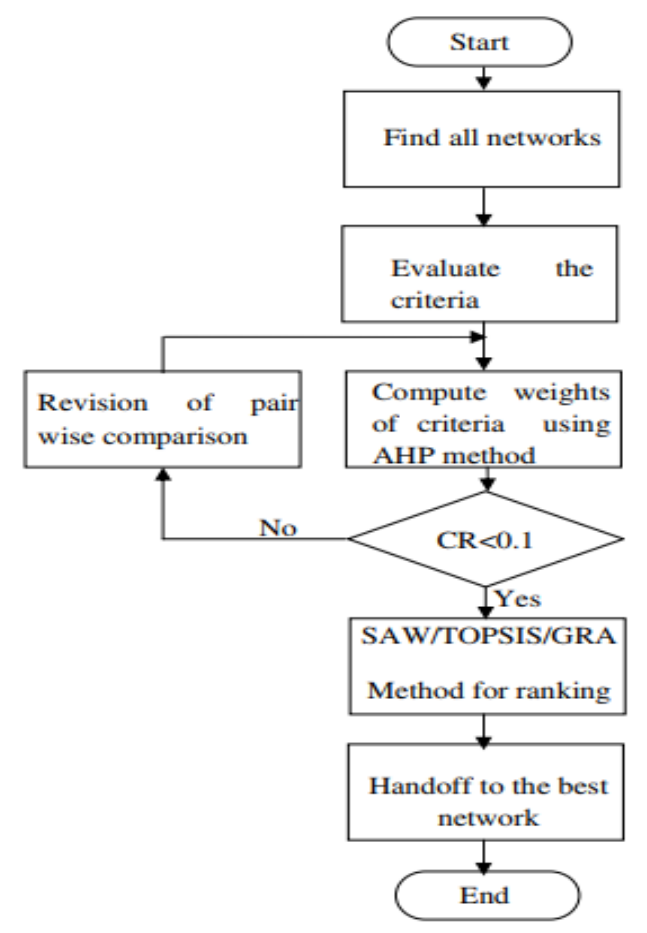

Fig. 7. Multi-Criterion Handover Decision Algorithm.

\section{A. Delay between Wi-Fi and WiMAX Networks with and without SDN}

Figure 8 illustrates the network delay by $\mathrm{WiFi}$ and WiMAX scenarios with and without SDN using OMNeT++4.6, In WiMAX, and WiFi, they represent the endto-end delay of all packets received by WiMAX or WiFi MACs of all nodes of the network, and they transmit to the upper layer.

According to the following figure, we observe that WiMAX does not require more delay, compared to WiFi to make the transfer from WiMAX to WiMAX or vice versa, compared to $\mathrm{WiFi}$, This is quite logical because the WiMAX network has been designed primarily to connect a very large number of users with high bandwidth with a minimum of time. 


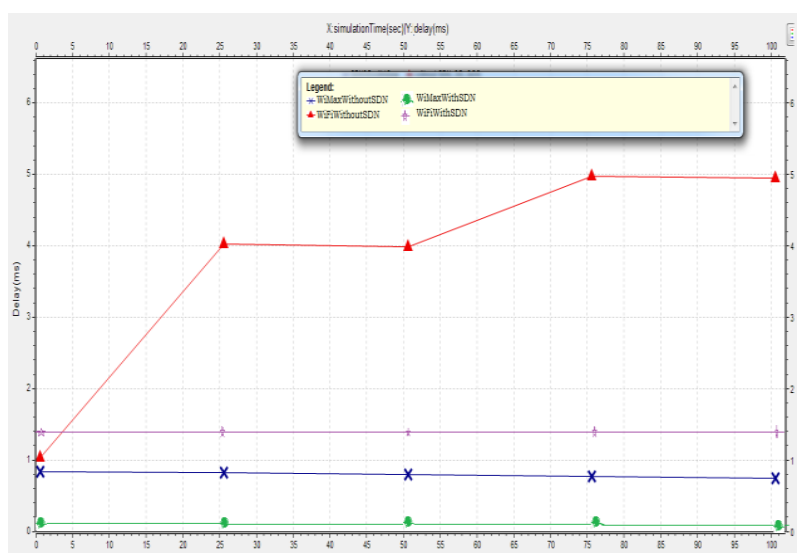

Fig. 8. Time delay between WiFi and WiMAX Networks with and without SDN.

\section{B. Throughout between WiFi and WiMAX Networks with and without SDN}

WiMAX is intended to connect a large number of clients, that's why we find its speed is higher or without SDN and more scalable with the implementation the SDN. As a results (Figure 9), WiMAX remains the most scalable compared to WiFi network with and without SDN.

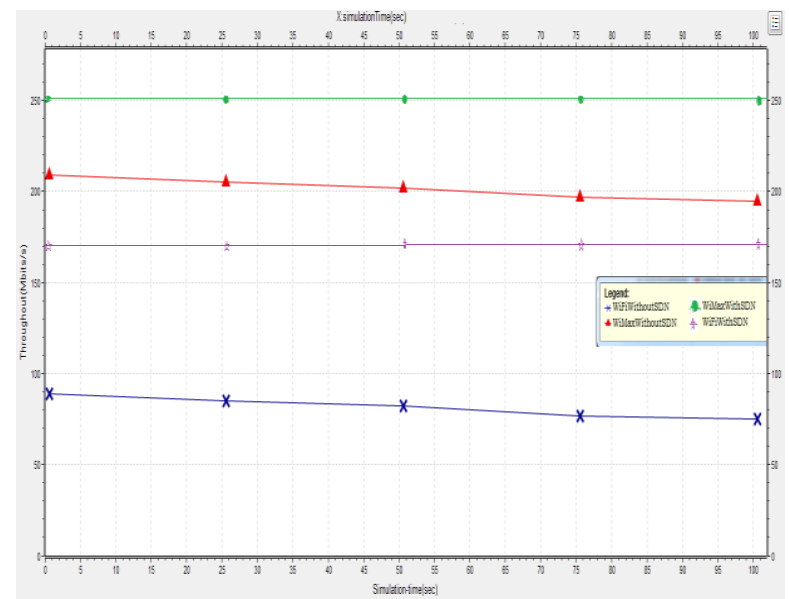

Fig. 9. Throughout between WiFi and WiMAX Networks with and without SDN.

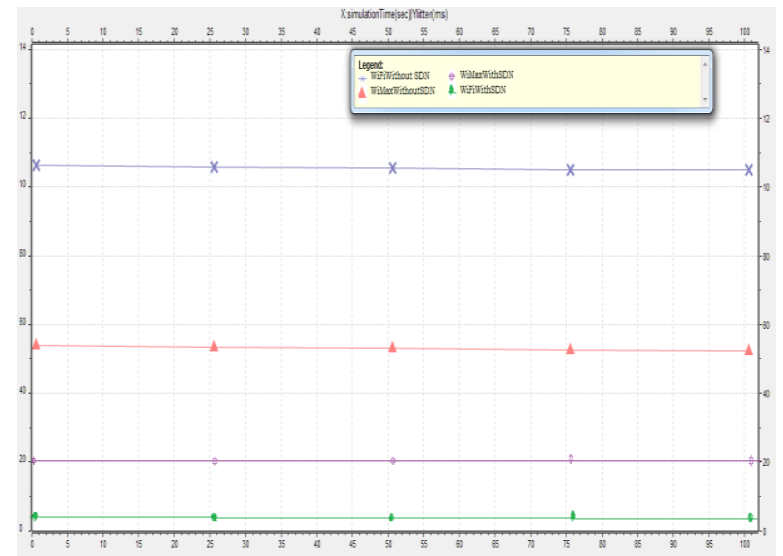

Fig. 10. Jitter between WiFi and WiMAX Networks with and without SDN.

\section{Jitter between WiFi and WiMAX Networks with and without SDN}

The results in Figure 10 shows that the jitter presented by WiFi and WiMAX scenarios with SDN is low compared to without SDN which requires more jitters using OMNeT++4.6, which justifies the impact of the addition of SDN is important.

\section{CONCLUSION}

This article presents performance improvement and evaluation of vertical handover between the WIFI and WiMAX by the implementation of a new algorithm at the level of the SDN controller which allows the handover decision by RSS, bit rate, jitter, and estimated delay using OMNeT++4.6. This algorithm is better placed to anticipate and initiate the transfer before it is required. This would include identifying and configuring wireless and TCP/ IP connections to the next access point before the actual handshake process.

\section{REFERENCES}

[1] Jeffrey G. Andrews, Arunabha Ghosh, Rias Muhamed "Fundamentals of WiMAX Understanding Broadband Wireless Networking', 2007

[2] Jolly Parikh, Anuradha Basu, "LTE Advanced: The 4G Mobile Broadband Technology", International Journal of Computer Applications (0975 - 8887) Volume 13- No.5, January 2011

[3] Fèten RIDENE RAISSI, et Adel RAISSI, "Authentification dans les Réseaux Wifi par le protocole radius", Thesis, 2010

[4] Gamal Abdel , Fadeel Mohamed Khalaf, Hesham Zarief Badr, "A comprehensive approach to vertical handoff in heterogeneous wireless networks", Journal of King Saud University - Computer and Information Sciences (2013) 25, 197-205, November 2012

[5] Slavisa Tomic, Marko Beko, Rui Dinis, "RSS-Based Localization in Wireless Sensor Networks Using Convex Relaxation: Noncooperative and Cooperative Schemes", IEEE Transactions on Vehicular Technology, Volume: 64, Issue: 5 , May 2015

[6] R. Les Cottrell, Saad Ansari, Parakram Khandpur, Ruchi Gupta, Richard Hughes-Jones, Michael Chen, Larry McIntosh, Frank Leers, "Characterization and evaluation of TCP and UDP-based transport on real networks", Volume 61, Issue 1-2, pp 5-20, February 2006

[7] Seok-Yee Tang, Peter Muller, Hamid R. Sharif, "WiMAX Security and Quality of Service, A John Wiley and Sons, Ltd., Publication, 2007

[8] Hung-Yu Wei ; S. Ganguly ; R. Izmailov ; Z.J. Haas, "Interferenceaware IEEE 802.16 WiMAX mesh networks", 2005 IEEE 61st Vehicular Technology Conference, June 2005

[9] Mojtaba Seyedzadegan, and Mohamed Othman, "IEEE 802.16: WiMAX Overview, WiMAX Architecture", International Journal of Computer Theory and Engineering, Vol. 5, No. 5, DOI: 10.7763/IJCTE.2013.V5.796, October 2013

[10] Haihong Zheng, Shashikant Maheshwari, Basavaraj Patil, Srinivas Sreemanthula, "Framework for internetworking between WMAN and WLAN networks", 2008

[11] Paul Mühlethaler, "802.11 et les réseaux sans fil”, book, EAN13 : 9782212111545, 2002

[12] Wen-Hsing Kuo, Wanjiun Liao, Tehuang Liu, “Adaptive Resource Allocation for Layer-Encoded IPTV Multicasting in IEEE 802.16 WiMAX Wireless Networks", IEEE Transactions on Multimedia, Volume: 13 , Issue: 1 , Feb. 2011

[13] Mohamed El Mahdi Boumezzough, Noureddine Idboufker, Abdellah Ait Ouahman, "Evaluation of SIP Call Setup Delay for VoIP in IMS", IEEE International Conference on Advanced Infocomm Technology, ICAIT 2012: Advanced Infocomm Technology pp 16-24, 2012

[14] Fatima LAASSIRI, Mohamed MOUGHIT, Noureddine IDBOUFKER, "Evaluation of the QoS parameters in different SDN architecture using OMNeT 4.6++", 2017 18th International Conference on Sciences and 
Techniques of Automatic Control and Computer Engineering (STA), 2017

[15] Raghunath Deshpande, "Overview of Different Approaches for Leveraging SDN in Mobile Networks", https://www.researchgate.net/publication/282322426, February 2015

[16] B. Wijnen, R. Presuhn, K. McCloghrie, "View-based Access Control Model (VACM) for the Simple Network Management Protocol (SNMP)", Network Working Group, Copyright (C) The Internet Society (2002)

[17] Anders Moberg, Dmitry M. Sonechkin, Karin Holmgren, Nina M. Datsenko, Wibjörn Karlén, "Highly variable Northern Hemisphere temperatures reconstructed from low- and high-resolution proxy data", Nature volume433, pages613-617, February 2005

[18] Honglin $\mathrm{Hu}$; Hsiao-Hwa Chen; Peter Mueller; Rose Qingyang $\mathrm{Hu}$; Yun Rui , "Software defined wireless networks (SDWN): Part 1 [Guest Editorial]", IEEE Communications Magazine, Volume: 53, Issue: 11 , November 2015

[19] Soujanya Bhumkar, Mayank Mehta, Josh Schwarzapel, Austin Shoemaker, "Method and system for displaying photos, videos, RSS and other media content in full-screen immersive view and grid-view using a browser feature", 2007
[20] Charles E. Perkins, “Ad Hoc Networking", CE Perkins - 2001 academia.edu [21] M.E. Baran, F.F. Wu, "Network reconfiguration in distribution systems for loss reduction and load balancing", IEEE Transactions on Power Delivery, Volume: 4 , Issue: 2 , Apr 1989

[21] Niranjan Suri, Jeffrey M. Bradshaw, Maggie R. Breedy, Paul T. Groth, Gregory A. Hill, Renia Jeffers, Timothy S. Mitrovich, Brian R. Pouliot, David S. Smith, "toward a strong and safe mobile agent system", ISBN:1-58113-230-1, Barcelona, Spain - June 03 - 07, 2000

[22] Mona Laroussi, Alain Derycke, Trigone-CIREL, "new e-learning services based on mobile and ubiquitous computing: Ubi-learn project", International Conference on Computer Aided Learning in Engineering education, 16-18 février 2004, 2004, Grenoble, France. 6 p., 2004.

[23] Zdenek Becvar, Jan Zelenka "Handovers in the Mobile WiMAX",https://www.researchgate.net/profile/Zdenek_Becvar/publicat ion/229049396_Handovers_in_the_Mobile_WiMAX/links/00b7d51521 9902288b000000.pdf 2006

[24] Niranjan Suri, Jeffrey M. Bradshaw, Maggie R. Breedy, Paul T. Groth, Gregory A. Hill, Renia Jeffers, "Strong Mobility and Fine-Grained Resource Control in NOMADS", LNCS, volume 1882, , pp. 2-15, Springer-Verlag Berlin Heidelberg, 2000 